\title{
MiR-29c-3p inhibits epithelial-mesenchymal transition to inhibit the proliferation, invasion and metastasis of cervical cancer cells by targeting SPARC
}

\author{
Ting Zou ${ }^{1,2,3}$, Yan $\mathrm{Gao}^{2}$, Mingrong $\mathrm{Qie}^{1,3}$ \\ ${ }^{1}$ Department of Gynecology and Obstetrics, West China Second University Hospital, Sichuan University, Chengdu, China; ${ }^{2}$ Department of \\ Gynecology, Guizhou Provincial People's Hospital, Guiyang, China; ${ }^{3}$ Key Laboratory of Obstetrics and Gynecologic and Pediatric Diseases and \\ Birth Defects of Ministry of Education, West China Second Hospital, Sichuan University, Chengdu, China \\ Contributions: (I) Conception and design: T Zou, M Qie; (II) Administrative support: M Qie; (III) Provision of study materials or patients: T Zou, Y \\ Gao; (IV) Collection and assembly of data: T Zou, Y Gao; (V) Data analysis and interpretation: T Zou, M Qie; (VI) Manuscript writing: All authors; \\ (VII) Final approval of manuscript: All authors. \\ Correspondence to: Mingrong Qie. Department of Gynecology and Obstetrics, West China Second University Hospital, Sichuan University; Key \\ Laboratory of Obstetrics and Gynecologic and Pediatric Diseases and Birth Defects of Ministry of Education, West China Second Hospital, Sichuan \\ University, Chengdu, China. Email: xmrjzz@126.com.
}

Background: Cervical cancer is one of the most common gynecological malignancies. Cancer recurrence and the poor efficacy of cervical cancer treatments are mainly caused by invasion and metastasis of cervical cancer cells. This study is to investigate whether miR-29c-3p can inhibit epithelial-mesenchymal transition (EMT) by targeting secreted protein acidic and rich in cysteine (SPARC), thus inhibiting the invasion and metastasis of human cervical cancer cells.

Methods: The expression levels of miR-29c-3p and SPARC in cervical cancer tissues and non-tumor adjacent tissues, human normal cervical epithelial cell line Ect1/E6E7 and human cervical cancer cell lines HeLa, CaSki, C-33A, HT-3 and SiHa were detected. After the expression of miR-29c-3p and SPARC was intervened in C-33A and SiHa cells, RT-qPCR was used to detect the expression levels of miR-29c-3p and SPARC. Western blot was performed to observe the expression levels of SPARC and EMT-related proteins. The proliferation rate of $\mathrm{C}-33 \mathrm{~A}$ and $\mathrm{SiHa}$ cells was measured using an MTT assay. The viability of the cells was determined using a cell colony formation assay. Apoptosis and cell cycle was measured using flow cytometry, and migration ability was observed using a wound healing assay. A transwell invasion assay was used to determine the invasion ability of the cells, whilst a dual-luciferase reporter assay verified that SPARC was a target gene of miR-29c-3p.

Results: miR-29c-3p was expressed at low levels in cervical cancer tissues and cells, while SPARC expression was upregulated. The luciferase reporter assay confirmed that miR-29c-3p targeted and bound to SPARC. MiR-29c-3p overexpression significantly inhibited the proliferation, invasion, migration, and cell cycle of cervical cancer cells, but promoted apoptosis. In the miR-29c-3p group (miR-29c-3p overexpression), EMT progression was inhibited by upregulating E-cadherin expression and downregulating $\mathrm{N}$-cadherin, vimentin, and Snail expression, which was contrary to the results of the in-miR-29c-3p group (inhibition of miR-29c-3p expression). In the miR-29c-3p + SPARC group (miR-29c-3p overexpression + SPARC overexpression), the effect of miR-29c-3p overexpression on cervical cancer cell functions was reversed.

Conclusions: miR-29c-3p can inhibit EMT by targeting SPARC, so as to inhibit the invasion and metastasis of cervical cancer cells.

Keywords: Cervical cancer; miR-29c-3p; secreted protein acidic and rich in cysteine (SPARC); epithelialmesenchymal transition (EMT) 
Submitted Sep 04, 2020. Accepted for publication Nov 16, 2020.

doi: 10.21037/atm-20-7272

View this article at: http://dx.doi.org/10.21037/atm-20-7272

\section{Introduction}

Cervical cancer is one of the most common gynecological malignancies, with approximaately 500,000 cases each year worldwide, $85 \%$ of which are in developing countries. At present, cervical cancer ranks as the highest incidence malignancy in Chinese women (1). Cancer recurrence and the poor efficacy of cervical cancer treatments are mainly caused by invasion and metastasis of cervical cancer cells (2). It has been reported that epithelial-mesenchymal transition (EMT) is closely related to the development of cervical cancer, and is one of the main causes of poor prognosis in patients with this disease $(3,4)$. EMT, characterized by the loss of epithelial features and gain of mesenchymal features, induces epithelial cells to migrate and invade, thus being involved in many pathophysiological processes including embryogenesis, organ differentiation, tissue inflammation, and wound healing $(5,6)$.

Secreted protein acidic and rich in cysteine (SPARC, also known as osteocalcin or BM40), mainly regulates the assembly and deposition of the extracellular matrix (ECM), as well as the signal transduction of growth factors. SPARC is expressed in bone, endothelial cells, and embryonic tissues, and is involved in the development and differentiation of chondrocytes and megakaryocytes $(7,8)$. In recent years, SPARC has been found to be associated with a highly aggressive tumor phenotype (9), as SPARC overexpression promotes the migration and invasion of breast cancer cells (10), and the postoperative survival rate is poor in non-small cell lung cancer patients with positive SPARC expression (11). However, SPARC expression depends on the type of cancer. SPARC is expressed at low levels in pancreatic cancer (12), colorectal cancer (13), and acute myeloid leukemia (14). Interestingly, SPARC expression in cervical cancer has not yet been reported.

MicroRNAs (miRNAs) refer to small non-coding RNA families of 21-25 nt in length which can recognize specific target mRNAs $(15,16)$. MiR-29c is a tumor suppressor miRNA, and its downregulated expression has been found in various cancers such as breast, lung, gastric, and liver cancer (17-19). SPARC, in turn, was found to be a direct target of miR-29c-3p (20). This study investigated the expression level of miR-29c-3p in cervical cancer and its effect on the biological function of cervical cancer cells, so as to find the molecular markers that effectively inhibit cervical cancer metastasis. Our study provides a novel approach to basic and clinical research into cervical cancer, and has unveiled new strategies for gene targeted therapy. We present the following article in accordance with the MDAR reporting checklist (available at http://dx.doi.org/10.21037/atm-207272).

\section{Methods}

\section{Clinical specimens}

Cervical cancer tissues (tumor tissues) and non-tumor adjacent tissues (normal tissues) were collected from cervical cancer patients treated in our hospital between June 2015 and June 2018. The study was conducted in accordance with the Declaration of Helsinki (as revised in 2013). The collection of clinical samples was approved by the Ethics Committee of Guizhou Provincial People's Hospital and was conducted in accordance with relevant regulations. All patients signed the informed consent and the agreement for specimen usage.

\section{Cell culture and transfection}

Human normal cervical epithelial cell line Ect1/E6E7 and human cervical cancer cell lines HeLa, CaSki, C-33A, HT-3, SiHa were purchased from the ATCC cell bank (Rockefeller, Maryland, USA). The cells were cultured in DMEM medium (Gibco, Grand Island, USA) containing $10 \%$ fetal bovine serum (FBS), $100 \mathrm{U} / \mathrm{mL}$ penicillin, and $100 \mu \mathrm{g} / \mathrm{mL}$ streptomycin, and were incubated at $37^{\circ} \mathrm{C}$ with $5 \% \mathrm{CO}_{2}$.

MiR-29c-3p mimics, an miR-29c-3p inhibitor, and their negative controls (NC) were purchased from GenePharma (Shanghai, China). The SPARC overexpression vector pcDNA3.1-SPARC and the empty vector pcDNA3.1 were synthesized by RiboBio (RiboBio Co., LTD, Guangzhou, China). C-33A and SiHa cells were transfected using Lipofectamine ${ }^{\mathrm{TM}} 3000$ in accordance with the reagent instructions. They were then divided into six groups: mimics NC (mi-NC) group, miR-29c-3p mimics (miR- 
$29 \mathrm{c}-3 \mathrm{p})$ group, inhibitor NC (in-NC) group, miR-29c-39 inhibitor (in-miR-29c-3p) group, miR-29c-3p mimics and empty vector pcDNA3.1 (miR-29c-3p + oe-NC) group, miR-29c-3p mimics and pcDNA3.1-SPARC (miR-29c-3p + SPARC) group.

\section{MTT assay}

At 24, 48 and 72 hours after transfection, the MTT reaction solution $(5 \mathrm{mg} / \mathrm{mL})$ was added to each well $(20 \mu \mathrm{L} /$ well $)$. After incubation at $37^{\circ} \mathrm{C}$ for 3 hours, the supernatant was aspirated. Next, $200 \mu \mathrm{L}$ dimethyl sulfoxide (DMSO) was added to each well and the solution was shaken for 2 minutes. The absorption value was assessed at $490 \mathrm{~nm}$ using an automatic microplate reader.

\section{Colony formation assay}

After transfection, the cells in each group were digested with trypsin to obtain cell suspensions. Subsequently, the cells were inoculated in 6-well plates (500 cells/well) and incubated at $37{ }^{\circ} \mathrm{C}$ with $5 \% \mathrm{CO}_{2}$ for colony formation. After 14 days of culture, the cells were fixed with $4 \%$ paraformaldehyde and stained with $0.1 \%$ crystal violet for $20 \mathrm{~min}$. Finally, the number of colony formations was calculated.

\section{Flow cytometry (FCM)}

At 48 hours after transfection, $1 \times 10^{5}$ cells were collected from each group and stained with propidium iodide (PI) and Annexin V-FITC binding solution. After rinsing twice with PBS, cells were collected to detect apoptosis using a flow cytometer (FACSCanto TM II, BD Biosciences, USA).

Furthermore, $1 \times 10^{6}$ cells were collected and incubated overnight in precooled $70 \%$ ethanol at $4{ }^{\circ} \mathrm{C}$. The next day, cells were stained with PI after RNaseA treatment and incubated at room temperature for 30 minutes. Finally, the cell cycle was analyzed using FCM.

\section{Transwell invasion assay}

Matrigel gel (Becton, Dickinson and Company, USA) was added to the upper chamber of the transwell. The transfected cell suspensions of each group were inoculated on the matrigel, while $500 \mu \mathrm{L}$ of complete medium was added to the lower chamber. After 48 hours of culture, the transwell chamber was taken out and the invasive cells were rinsed 3 times with PBS, fixed with 4\% paraformaldehyde for 10 minutes, and stained with crystal violet solution. Finally, 5 fields were randomly selected under a microscope for imaging and analysis.

\section{Wound healing assay}

After transfection, the cells in each group were inoculated into 6-well plates. When the confluency reached 90\%, a small pipette tip was used to scratch vertically and PBS was used to rinse the cells. Subsequently, the cells were added to serum-free medium and cultured at $37{ }^{\circ} \mathrm{C}$ with $5 \% \mathrm{CO}_{2}$ and constant temperature. The migration process was captured under an inverted microscope at 0 hours (baseline) and 24 hours.

\section{$R T-q P C R$}

Total RNA was extracted from clinical tissues and cells using TRIzol reagent or the miRNANeasy Mini Kit (Qiagen, Germany) according to the manufacturer's instructions. The RNA was reverse transcribed into cDNA, and the RT-qPCR reaction was performed with 40 cycles using the following parameters: $90^{\circ} \mathrm{C}$ for 3 minutes, $95^{\circ} \mathrm{C}$ for 15 seconds and $60{ }^{\circ} \mathrm{C}$ for 30 seconds. U6 and GAPDH were selected as internal reference genes, and the relative expression was calculated using the $2^{-\Delta \Delta \mathrm{Ct}}$ method. The experiment was repeated 3 times. The primer sequences used in this study were shown in Table 1 .

\section{Western blot}

The transfected cells were collected, and total protein was extracted using RIPA lysis buffer. After the protein concentration was determined, the protein was first separated using SDS-PAGE then electrophoretically transferred to a polyvinylidene difluoride (PVDF) membrane. Following the blocking step with $5 \%$ skim milk power for 1 hour at room temperature, the membrane was incubated overnight at $4{ }^{\circ} \mathrm{C}$ with the following primary antibodies: SPARC (Abcam, UK), E-cadherin (Abcam, UK), Vimentin (Abcam, UK), N-cadherin (Abcam, UK), Snail (Abcam, UK), and GAPDH (Abcam, UK). GAPDH was used as an internal control. After rinsing with PBST, membranes were incubated with secondary antibodies at room temperature for 1 hour, then rinsed with PBST. The target proteins were developed by chemiluminescence, and the images were analyzed quantitatively using Image $\mathbf{J}$ 
Table 1 RT-qPCR primer sequences

\begin{tabular}{lll}
\hline Gene & Primer & Sequences $\left(5^{\prime}-3^{\prime}\right)$ \\
\hline miR-29c-3p & Forward & ACACTGCTCCAGGGTAGCACCATTTGAAT \\
& Reverse & TGGTGTCGTGGAGTCG \\
F6 & Forward & CTCGCTTCGGCAGCACA \\
RPARC & Reverse & AACGCTTCACGAATTTGCGT \\
& Forward & GCTGTTGGAAACGGATTG \\
GAPDH & Reverse & CTTGCCATGTGGGTTCTGACT \\
& Forward & AACGGATTTGGTCGTATTG \\
& Reverse & GGAAGATGGTGATGGGATT \\
\hline
\end{tabular}

software (National Institutes of Health, USA).

\section{Dual-luciferase reporter assay}

SPARC was predicted to be a target gene of miR-29c-3p using TargetScan, Starbase V2.0, and miRTarBase, and their binding was verified using a luciferase reporter assay. The 3'-untranslated region (UTR) of the SPARC gene was cloned into the pmirGLO vector, and a mutated sequence was generated and cloned into the vector. The reporter plasmids SPARC-wild-type (WT) and SPARC-mutant (MUT) were constructed by RiboBio. At 48 hours after the transfection with miR-29c-3p mimics and SPARC-WT or SPARC-MUT, luciferase activity was detected using a dualluciferase reporter assay.

\section{Statistical analysis}

SPSS 19.0 was used for statistical analysis, and GraphPad Prism 5 software was used for graphing. Experimental data were all expressed as mean \pm standard deviation (SD). Comparisons between two groups were performed using $t$-tests, and comparisons of multiple groups were performed using one-way analysis of variance (ANOVA). $\mathrm{P}<0.05$ indicated a significant difference.

\section{Results}

MiR-29c-3p expression is downregulated in cervical cancer tissues and cells

The results of RT-qPCR showed that miR-29c-3p was expressed at low levels in tumor tissues compared to normal tissues (Figure 1A). Furthermore, the expression of miR- 29c-3p was significantly downregulated in $\mathrm{HeLa}, \mathrm{CaSki}$, C-33A, HT-3, and SiHa cell lines compared to the Ect1/ E6E7 cell line, with the lowest expression in the C-33A and $\mathrm{SiHa}$ cell lines (Figure 1B). The results of western blotting revealed that the expression of the EMT marker protein E-cadherin was significantly decreased, while the expression levels of vimentin, $\mathrm{N}$-cadherin and Snail were significantly increased in tumor tissues $(\mathrm{P}<0.01)$ (Figure $1 C)$. These results confirmed that miR-29c-3p might be involved in the occurrence of cervical cancer, which is closely related to EMT. To further investigate the function of miR-29c-3p in cervical cancer cells, we transfected miR-29c-3p mimics or an miR-29c-3p inhibitor into C-33A and $\mathrm{SiHa}$ cells to upregulate or inhibit miR-29c-3p expression, respectively (Figure 1D,E).

\section{MiR-29c-3p inbibits proliferation and the cell cycle, and promotes apoptosis in cervical cancer cells}

The expression of miR-29c-3p in C-33A and SiHa cells was intervened to investigate cell proliferation, migration and invasion. As shown in Figure $2 A$, the proliferation rate of $\mathrm{C}-33 \mathrm{~A}$ and $\mathrm{SiHa}$ cells significantly reduced after upregulating miR-29c-3p expression, whilst the proliferation rate increased after inhibiting miR-29c$3 \mathrm{p}$ expression. A colony formation assay confirmed that following upregulation of miR-29c-3p, the number of C-33A and SiHa colonies significantly decreased, while the colonies significantly increased after downregulating miR29c-3p expression (Figure 2B).

FCM was carried out detect the apoptosis rate and cell cycle of C-33A and SiHa cells after transfection in the mi$\mathrm{NC}, \mathrm{miR}-29 \mathrm{c}-3 \mathrm{p}$, in-NC, and in-miR-29c-3p groups. As 

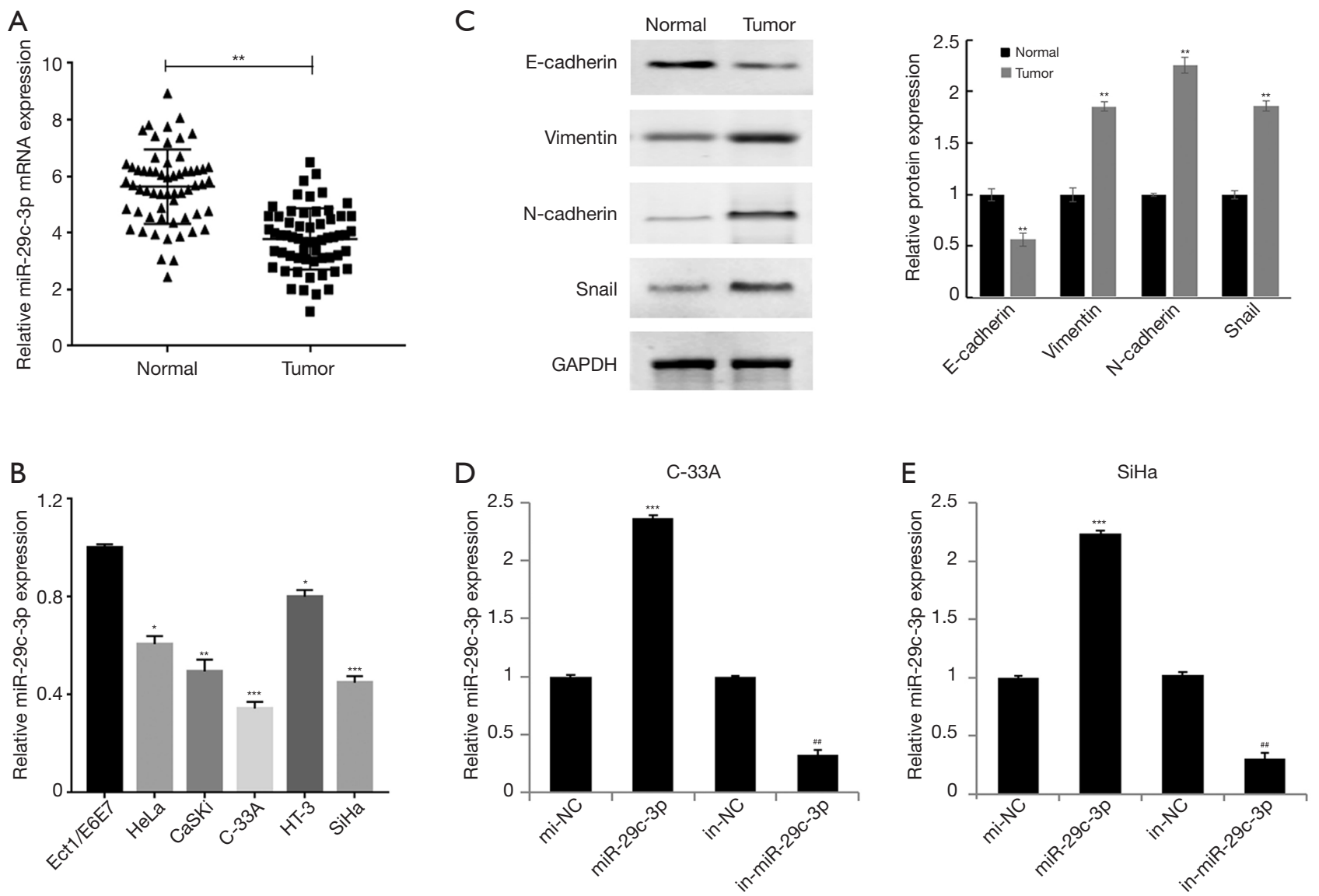

Figure 1 MiR-29c-3p was expressed at low levels in cervical cancer tissues and cells. RT-qPCR to detect the relative expression of miR-29c$3 \mathrm{p}$ in tumor tissues and normal tissues; (B) RT-qPCR to detect the expression of miR-29c-3p in Ect1/E6E7, HeLa, CaSki, C-33A, HT-3 and SiHa cell lines; (C) Western blot to detect the expression of EMT-related proteins in tumor tissues and normal tissues; (D) RT-qPCR to detect the expression of miR-29c-3p in C-33A cells after transfection with miR-29c-3p mimics or an inhibitor; (E) RT-qPCR to detect the expression of miR-29c-3p in SiHa cells after transfection with miR-29c-3p mimics or an inhibitor. ${ }^{*}, \mathrm{P}<0.05 ;{ }^{* *}, \mathrm{P}<0.01$; ***, $\mathrm{P}<0.001$ vs. control group; ${ }^{\#}, \mathrm{P}<0.01$ vs. in-NC group. GAPDH, glyceraldehyde-3-phosphate dehydrogenase.

shown in Figure $2 C$ and $D$, miR-29c-3p overexpression promoted cell apoptosis and cell cycle arrest in the G0/G1 phase, whilst inhibition of miR-29c-3p expression reduced cell apoptosis and increased the number of cells in $\mathrm{S}$ phase. The above results indicated that miR-29c-3p overexpression promoted apoptosis and inhibited division of cervical cancer cells.

\section{MiR-29c-3p inbibits invasion and migration of cervical cancer cells by inbibiting EMT}

Invasion and migration are important indicators to assess cancer cell metastasis. In this study, a transwell invasion assay and wound healing assay were performed to observe the effects of miR-29c-3p expression on the invasion and migration of C-33A and SiHa cells. As shown in Figure $3 \mathrm{~A}$, miR-29c-3p overexpression reduced cell invasion in C-33A and $\mathrm{SiHa}$ cells compared with the mi-NC group, whilst cell invasion was enhanced in the in-miR-29c-3p group compared with the in-NC group. As shown in Figure 3B, the wound field of cervical cancer cells was significantly wider and migration was weakened in the miR-29c$3 \mathrm{p}$ group, contrary to the results of the in $-\mathrm{miR}-29 \mathrm{c}-3 \mathrm{p}$ group. Western blot assay showed that the expression of E-cadherin was significantly upregulated, while the expression levels of vimentin, N-cadherin, and Snail were significantly decreased in the miR-29c-3p group (Figure 3C). The above results indicated that miR-29c- 

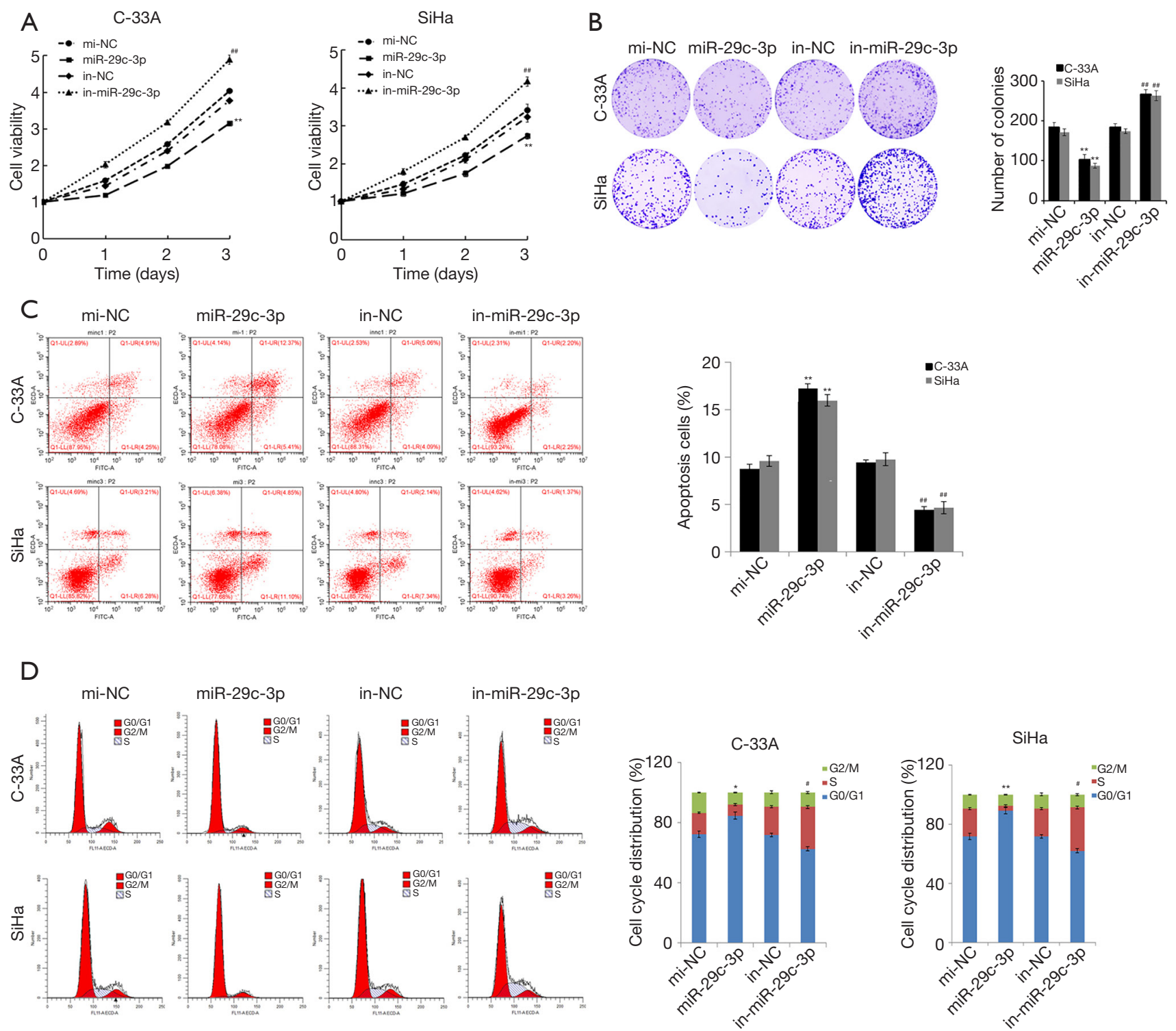

Figure 2 Effects of miR-29c-3p on proliferation, cell cycle, and apoptosis in cervical cancer cells. C-33A and SiHa cells were transfected with miR-29c-3p mimics and an miR-29c-3p inhibitor. (A) MTT assay to detect the proliferation of C-33A and SiHa cells; (B) colony formation assay to detect the colony formation ability of C-33A and SiHa cells; (C) flow cytometry (FCM) to detect the apoptosis rate of C-33A and SiHa cells; (D) FCM to detect the cell cycle of C-33A and SiHa cells. ${ }^{*}, \mathrm{P}<0.05$ and ${ }^{* *}, \mathrm{P}<0.01$ s. mi-NC group; ${ }^{*}, \mathrm{P}<0.05$ and ${ }^{\# \#}$, $\mathrm{P}<0.01$ vs. in-NC group.

$3 p$ inhibited the invasion and migration of cervical cancer cells by inhibiting EMT.

\section{MiR-29c-3p targets SPARC}

A binding site between miR-29c-3p and SPARC was predicted using bioinformatics software (Figure $4 A$ ). To verify whether miR-29c-3p targets SPARC, WT or MUT plasmids containing SPARC target sequences (SPARCWT group/SPARC-MUT group) were constructed. As shown in Figure 4B, in cervical cancer cells, mi-R-29c-3p mimics significantly downregulated luciferase activity in the SPARC-WT group, but had no significant effect on the SPARC-MUT group. Meanwhile, both mRNA and protein 


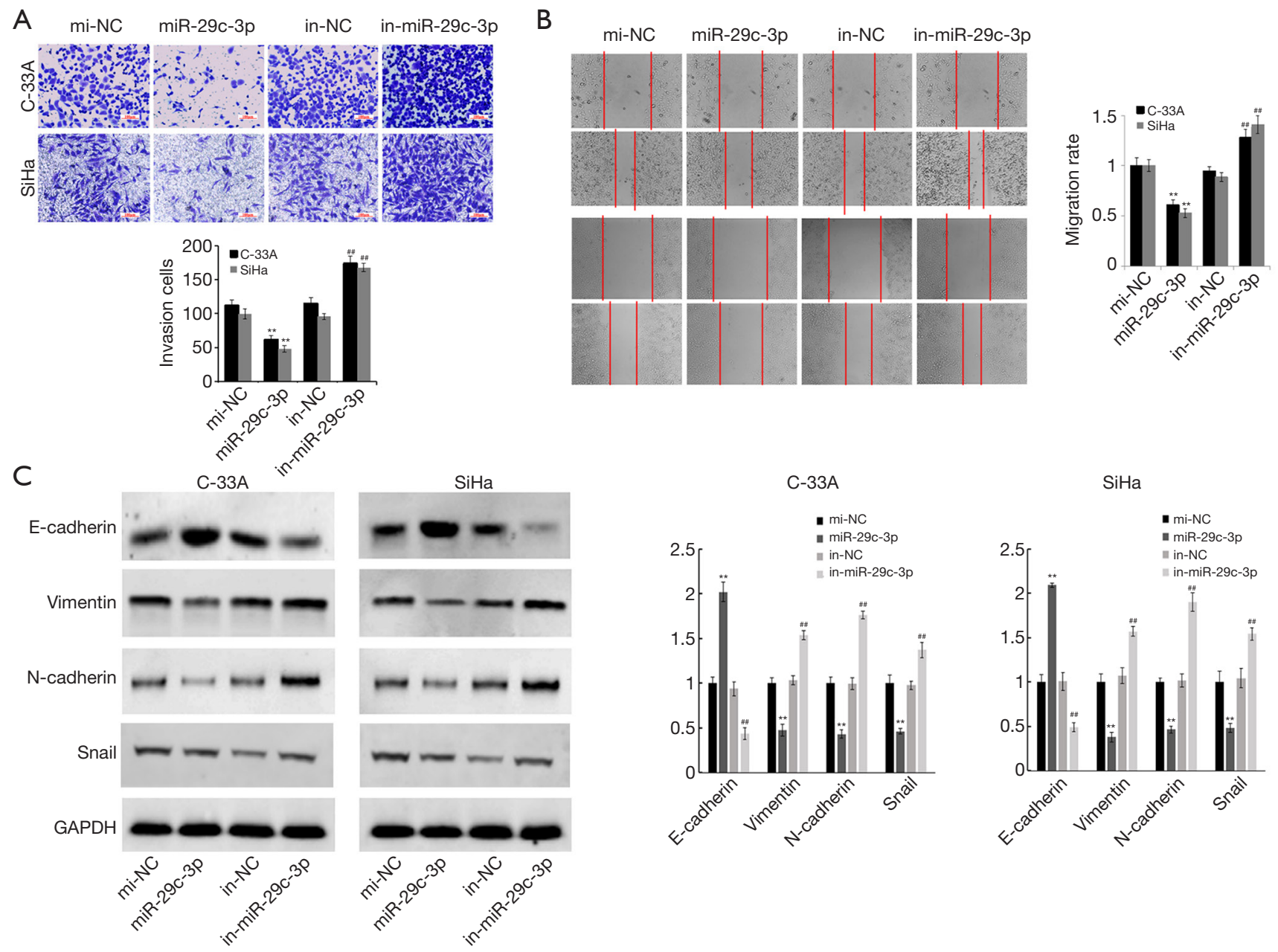

Figure 3 Effect of miR-29c-3p on the migration and invasion of cervical cancer cells, and the EMT process. C-33A and SiHa cells were transfected with miR-29c-3p mimics and an miR-29c-3p inhibitor. (A) Transwell invasion assay to detect the invasion of C-33A and SiHa cells, the scale of the map is 1:100 $\mu \mathrm{m} .0 .1 \%$ Crystal Violet Staining Solution: 100×; (B) wound healing assay to detect the migration of C-33A and SiHa cells; (C) Western blot to detect the expression of EMT-related proteins in C-33A and SiHa cells. ${ }^{* *}, \mathrm{P}<0.01$ vs. mi-NC group; ${ }^{\# \#}$, $\mathrm{P}<0.01$ vs. in-NC group. GAPDH, glyceraldehyde-3-phosphate dehydrogenase.

expression of SPARC were downregulated in C-33A and $\mathrm{SiHa}$ cells in the miR-29c-3p group, which was contrary to the results of the in-miR-29c-3p group (Figure 4C,D). These results confirmed that miR-29c-3p targeted and inhibited SPARC expression.

Furthermore, the results of RT-qPCR showed that SPARC mRNA was significantly upregulated in cervical cancer tissues and cell lines (Figure 4E,F). Correlation analysis revealed a negative correlation between miR$29 \mathrm{c}-3 \mathrm{p}$ and SPARC expression in cervical cancer tissues (Figure $4 G$ ). The above results indicated that miR-29c$3 \mathrm{p}$ bound specifically to SPARC and inhibited SPARC expression in cervical cancer tissues and cells.

\section{$M i R-29 c-3 p$ regulates the proliferation, invasion, migration, and EMT of cervical cancer cells by inbibiting SPARC expression}

A cell rescue assay was carried out to further determine whether miR-29c-3p mediated the function of cervical cancer cells by regulating SPARC. As shown in Figure $5 \mathrm{~A}$ and $B$, the expression of SPARC mRNA was significantly downregulated in C-33A and SiHa cells in the miR-29c-3p group, while the relative expression of SPARC mRNA was 
A

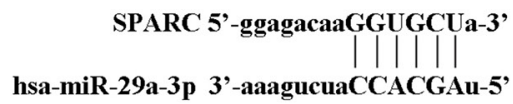

B

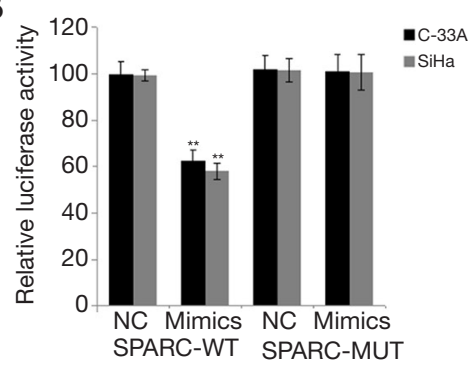

D

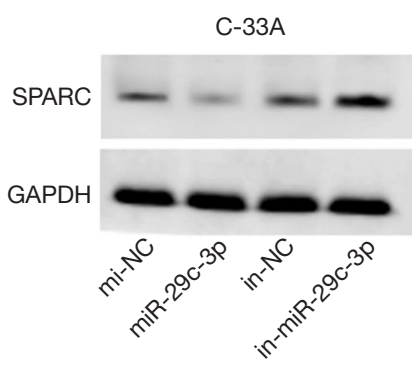

$E$

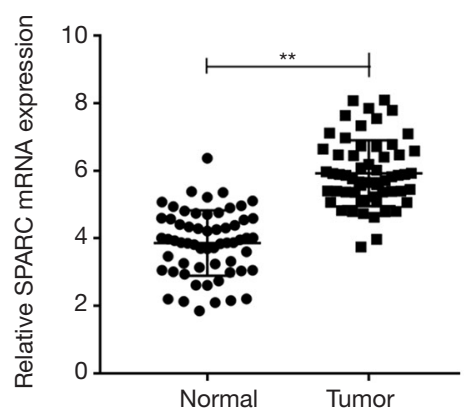

C

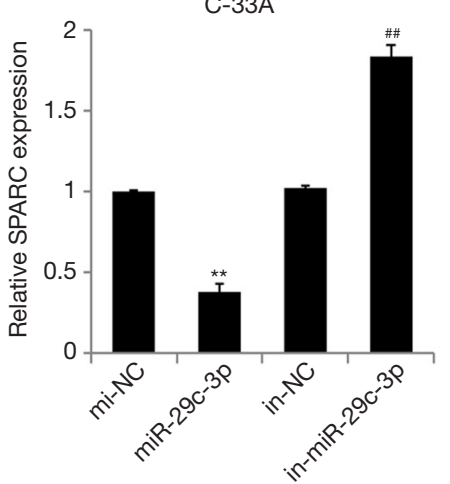

$\mathrm{SiHa}$

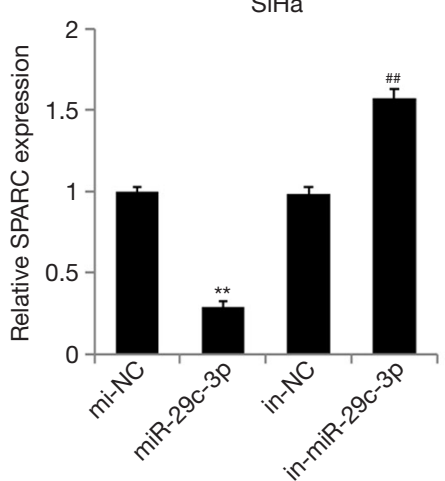

$\mathrm{SiHa}$

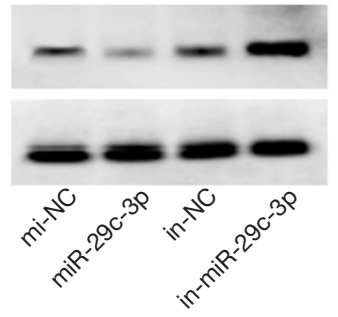

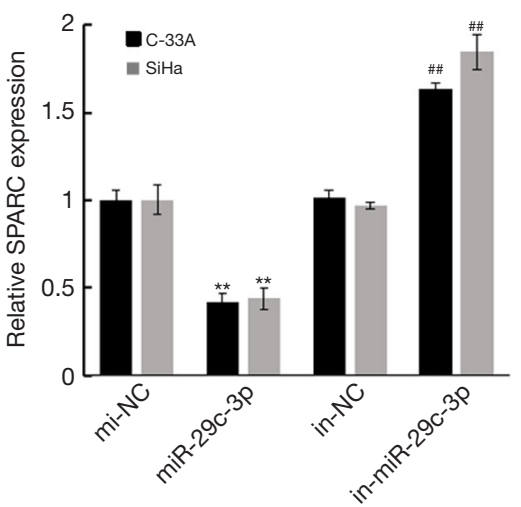

$\mathrm{F}$

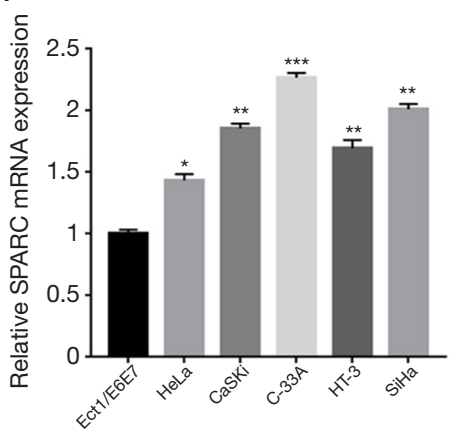

G

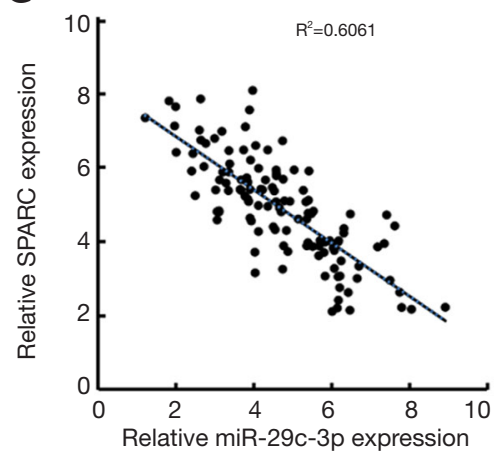

Figure 4 MiR-29c-3p targets SPARC in cervical cancer cells. (A) Bioinformatics software predicted the binding site between miR-29c$3 p$ and SPARC; (B) dual-luciferase reporter assay to detect the targeting relationship between miR-29c-3p and SPARC; (C) RT-qPCR to detect the expression of SPARC in C-33A and SiHa cells after transfection with miR-29c-3p mimics and an miR-29c-3p inhibitor; (D) Western blot to detect the expression of the SPARC protein; (E) RT-qPCR to detect the relative expression of SPARC in tumor tissues and normal tissues; (F) RT-qPCR to detect the relative expression of SPARC in Ect1/E6E7, HeLa, CaSki, C-33A, HT-3 and SiHa cell lines; (G) correlation analysis of miR-29c-3p and SPARC expression in tumor tissues. ${ }^{*}, \mathrm{P}<0.05$; ${ }^{* *}, \mathrm{P}<0.01$ and ${ }^{* * *}, \mathrm{P}<0.001$ vs. mi-NC group; \#, $\mathrm{P}<0.01$ vs. in-NC group. SPARC, secreted protein acidic and rich in cysteine; SPARC-WT, SPARC-wild-type; SPARC-MUT, SPARCmutant; GAPDH, glyceraldehyde-3-phosphate dehydrogenase. 

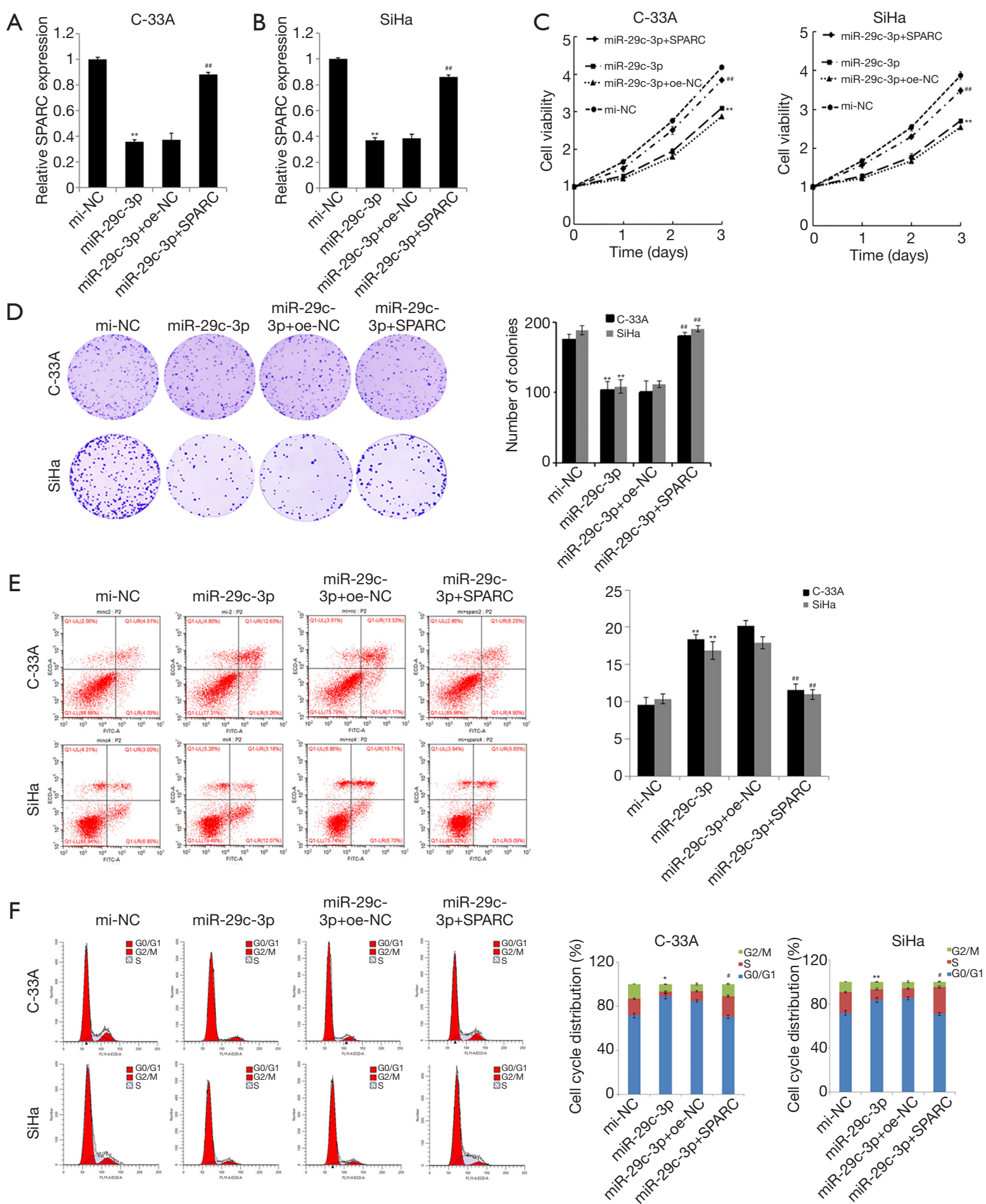

Figure $5 \mathrm{MiR}-29 \mathrm{c}-3 \mathrm{p}$ regulates the proliferation and apoptosis of cervical cancer cells by inhibiting SPARC. C-33A and SiHa cells were transfected with mi-NC, miR-29c-3p, miR-29c-3p + oe-NC, and miR-29c-3p + SPARC. (A,B) RT-qPCR to detect the relative expression of SPARC in C-33A cells (A) and SiHa cells (B); (C) MTT assay to detect the proliferation rate of C-33A and SiHa cells; (D) colony formation assay to detect cell colony formation ability; (E) FCM to detect the apoptosis rate of C-33A and SiHa cells; (F) FCM to detect the cell cycle of C-33A and SiHa cells. * $\mathrm{P}<0.05$ and ${ }^{* *}, \mathrm{P}<0.01$ vs. mi-NC group; ${ }^{*}, \mathrm{P}<0.05$ and ${ }^{\# \#}, \mathrm{P}<0.01$ vs. miR-29c-3p + oe-NC group. SPARC, secreted protein acidic and rich in cysteine. 
upregulated in the miR-29c-3p + SPARC group. The results of the MTT assay and colony formation assay showed that the proliferation of cervical cancer cells was reduced and the number of cell clones was reduced in the miR-29c-3p group, however, these parameters were increased in the miR-29c-3p + SPARC group (Figure 5C,D). The results of FCM showed that miR-29c-3p overexpression promoted apoptosis and inhibited the cell cycle, which was reversed in the miR-29c-3p + SPARC group (Figure $5 E, F$ ).

The results of the transwell invasion assay and wound healing assay confirmed that upregulation of miR-29c-3p expression reduced the invasion and migration of C-33A and $\mathrm{SiHa}$ cells, which was reversed in the miR-29c-3p + SPARC group (Figure $6 A, B)$. Finally, expression levels of EMTrelated proteins were detected, and the results showed that the expression of E-cadherin was significantly upregulated while the expression levels of vimentin, $\mathrm{N}$-cadherin, and Snail were significantly downregulated in C-33A and $\mathrm{SiHa}$ cells in the miR-29c-3p group. Contrastingly, the corresponding expression levels were reversed in the miR$29 c-3 p+$ SPARC group (Figure 6C). These results indicated that $\mathrm{miR}-29 \mathrm{c}-3 \mathrm{p}$ regulated the proliferation, invasion, migration and EMT of cervical cancer cells by inhibiting SPARC.

\section{Discussion}

MiRNAs are widely involved in the regulation of various functional genes. Functional disorders of miRNAs are often related to the development and metastasis of tumor cells (21). Recent studies have also found that miR-29c$3 p$ is associated with a variety of malignancies, showing abnormal expression in cancer cells (22). However, there are still few studies on miR-29c-3p in cervical cancer. Therefore, we hypothesized that miR-29c-3p also has specific physiological functions in cervical cancer. In this study, we found that the expression level of miR-29c-3p in cervical cancer cells was significantly lower than that in normal cells, indicating that the downregulated expression of miR-29c-3p is an important biomarker of cervical cancer. Invasion and migration directly reflect the metastatic ability of cancer cells, as Chen et al. (23) reported that miR-29c-3p inhibited the invasion and migration of colon cancer cells by targeting PHLDB2. In this study, it was found that miR$29 c-3 p$ overexpression significantly inhibited invasion and migration in cervical cancer cell using transwell invasion and wound healing assays. EMT is a physiological process in which epithelial cells lose their features but obtain features of mesenchymal cells. MiR-128 overexpression in melanoma cells was reported to inhibit the expression of N-cadherin, thereby inhibiting EMT and migration of melanoma cells (24). Therefore, we speculated that miR$29 c-3 p$ overexpression also inhibited EMT of cervical cancer cells. Through western blot, miR-129c-3p was observed to significantly increase E-cadherin expression while inhibiting the expression of vimentin, $\mathrm{N}$-cadherin, and Snail. The results indicate that miR-29c-3p inhibits EMT of cervical cells, thus inhibiting the invasion and migration of cervical cancer cells.

SPARC is a multifunctional secreted protein with various biological activities, and can specifically bind to miRNAs and participate in the regulation of several biological functions. Song et al. (25) reported that the members of the miR-29 family can regulate glucose metabolism by binding to SPARC. Furthermore, previous studies have found that hsa-miR-29c-3p can regulate the biological function of colorectal cancer cells by targeting SPARC (20). In this study, a specific binding site between miR-29c-3p and SPARC was predicted using bioinformatics software, and a dual-luciferase reporter assay confirmed that miR-29c$3 p$ bound to SPARC. Therefore, we speculated that miR$29 \mathrm{c}-3 \mathrm{p}$ regulated invasion and metastasis of cervical cancer cells by binding to SPARC. The results of quantitative experiments showed that the expression levels of SPARC mRNA and protein in cervical cancer cells were higher than those in normal cells, indicating that SPARC was important in cervical cancer cells. MiR-29c-3p overexpression was then able to inhibit the expression of SPARC, and correlation analysis showed a negative correlation between the two. Subsequently, a cell rescue assay was performed to further investigate how miR-29c-3p and SPARC affect cervical cancer cells together. The experimental results confirmed an antagonistic effect between miR-29c-3p and SPARC, hence, SPARC overexpression could counteract the inhibitory effect of miR-29c-3p overexpression on the migration, invasion and EMT of cervical cancer cells.

\section{Conclusions}

In summary, miR-29c-3p was expressed at low levels in cervical cancer tissues and cells. MiR-29c-3p can specifically bind to SPARC and negatively regulate SPARC expression, thus inhibiting the invasion and metastasis of cervical cancer cells by inhibiting EMT. This study provides insights into a molecular marker to inhibit cervical cancer metastasis, and new strategies for targeted therapy, providing a basis for 
A

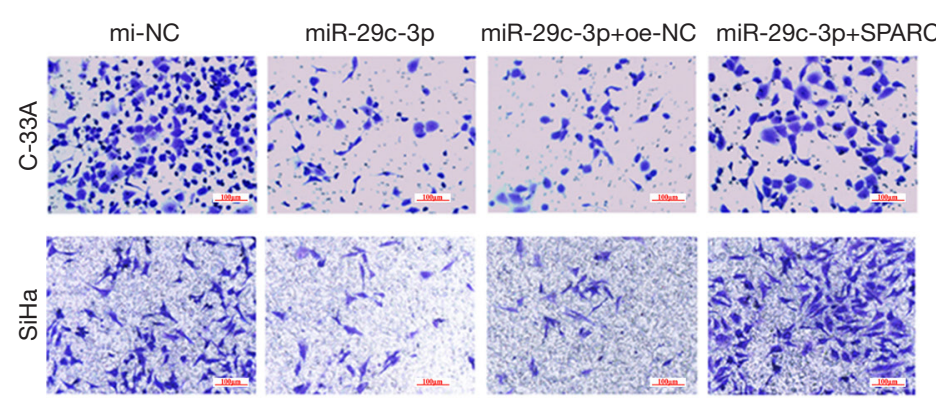

B

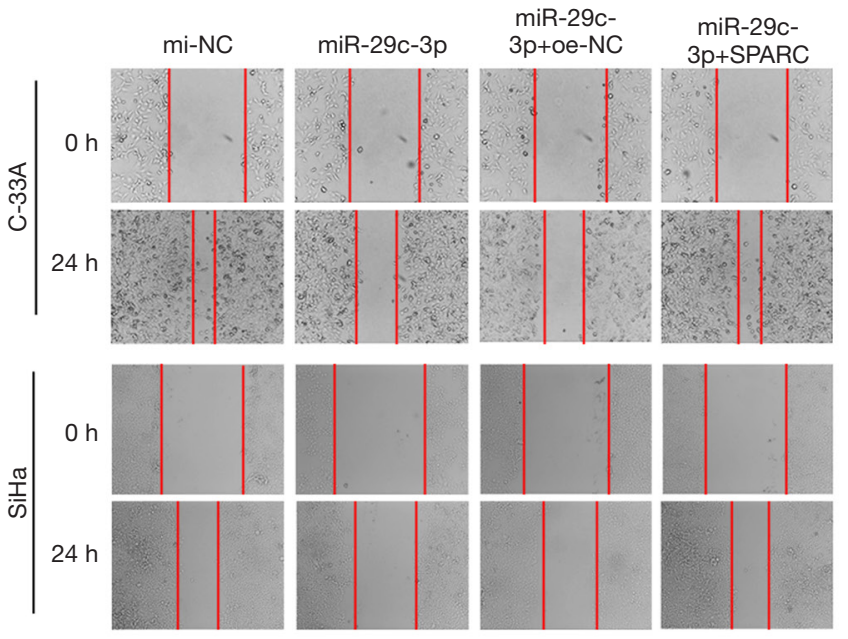

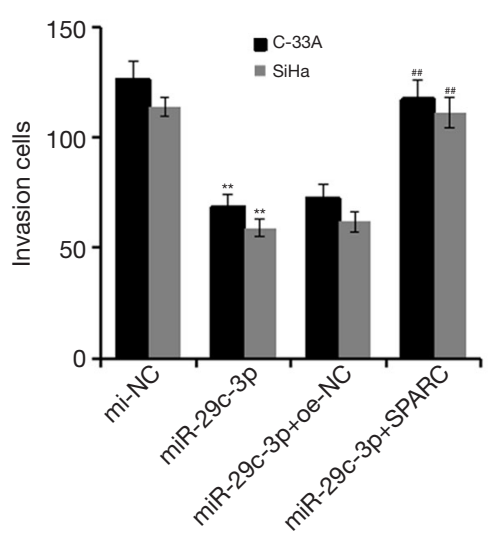

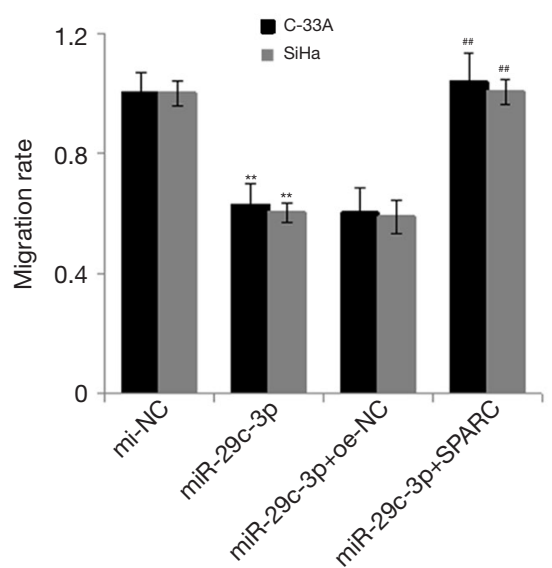

C

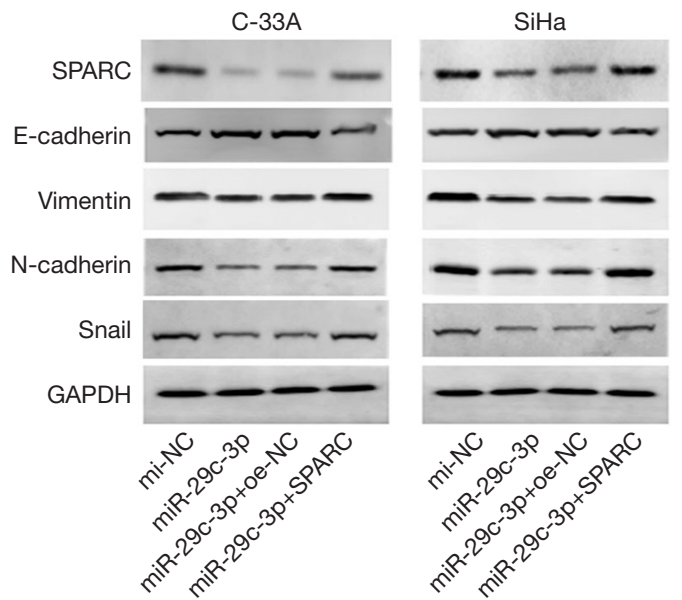

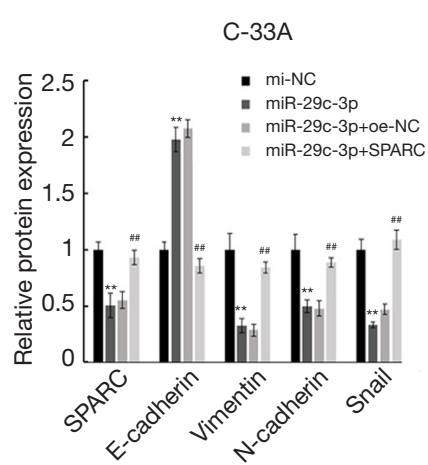

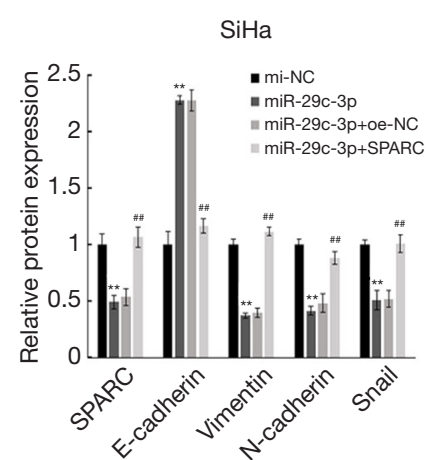

Figure 6 MiR-29c-3p regulates the invasion, migration, and EMT of cervical cancer by inhibiting SPARC. C-33A and SiHa cells were transfected with mi-NC, miR-29c-3p, miR-29c-3p + oe-NC and miR-29c-3p + SPARC. (A) Transwell invasion assay to detect the invasion ability of C-33A and SiHa cells, The scale of the map is 1:100 $\mu \mathrm{m}$. 0.1\% Crystal Violet Staining Solution: 100×; (B) wound healing assay to detect the migration ability of C-33A and SiHa cells; (C) Western blot to detect the expression levels of EMT-related proteins in C-33A and $\mathrm{SiHa}$ cells. **, $\mathrm{P}<0.01$ vs. mi-NC group; ${ }^{\# \#}, \mathrm{P}<0.01$ vs. miR-29c-3p + oe-NC group. SPARC, secreted protein acidic and rich in cysteine; GAPDH, glyceraldehyde-3-phosphate dehydrogenase. 
further basic and clinical research into cervical cancer.

\section{Acknowledgments}

Thanks go to Guangzhou Yujia Biotechnology Co., Ltd for their help in the field.

Funding: This work was supported by Youth Foundation of Guizhou Provincial People's Hospital (GZSYQN(2019)02).

\section{Footnote}

Reporting Checklist: The authors have completed the MDAR reporting checklist. Available at http://dx.doi.org/10.21037/ atm-20-7272

Data Sharing Statement: Available at http://dx.doi. org/10.21037/atm-20-7272

Conflicts of Interest: All authors have completed the ICMJE uniform disclosure form (available at http://dx.doi. org/10.21037/atm-20-7272). The authors have no conflicts of interest to declare.

Ethical Statement: The authors are accountable for all aspects of the work in ensuring that questions related to the accuracy or integrity of any part of the work are appropriately investigated and resolved. The study was conducted in accordance with the Declaration of Helsinki (as revised in 2013). The collection of clinical samples was approved by the Ethics Committee of Guizhou Provincial People's Hospital and was conducted in accordance with relevant regulations. All patients signed the informed consent and the agreement for specimen usage.

Open Access Statement: This is an Open Access article distributed in accordance with the Creative Commons Attribution-NonCommercial-NoDerivs 4.0 International License (CC BY-NC-ND 4.0), which permits the noncommercial replication and distribution of the article with the strict proviso that no changes or edits are made and the original work is properly cited (including links to both the formal publication through the relevant DOI and the license). See: https://creativecommons.org/licenses/by-nc-nd/4.0/.

\section{References}

1. Bray F, Ferlay J, Soerjomataram I, et al. Global cancer statistics 2018: GLOBOCAN estimates of incidence and mortality worldwide for 36 cancers in 185 countries. CA Cancer J Clin 2018;68:394-424.

2. Colombo N, Carinelli S, Colombo A, et al. Cervical cancer: ESMO clinical practice guidelines for diagnosis, treatment and follow-up. Ann Oncol 2012;23 Suppl 7:vii27-32.

3. Xu D, Liu S, Zhang L, Song L. MiR-211 inhibits invasion and epithelial-to-mesenchymal transition (EMT) of cervical cancer cells via targeting MUC4. Biochem Biophys Res Commun 2017;485:556-62.

4. Jiang Z, Song Q, Zeng R, et al. MicroRNA-218 inhibits EMT, migration and invasion by targeting SFMBT1 and DCUN1D1 in cervical cancer. Oncotarget 2016;7:45622-36.

5. Thiery JP, Acloque H, Huang RY, et al. Epithelialmesenchymal transitions in development and disease. Cell 2009;139:871-90.

6. Takai M, Terai Y, Kawaguchi H, et al. The EMT (epithelial mesenchymal-transition)-related protein expression indicates the metastatic status and prognosis in patients with ovarian cancer. J Ovarian Res 2014;7:76.

7. Nagaraju GP, Sharma D. Anti-cancer role of SPARC, an inhibitor of adipogenesis. Cancer Treat Rev 2011;37:559-66.

8. Podhajcer OL, Benedetti LG, Girotti MR, et al. The role of the matricellular protein SPARC in the dynamic interaction between the tumor and the host. Cancer Metastasis Rev 2008;27:691-705.

9. Tai IT, Tang MJ. SPARC in cancer biology: its role in cancer progression and potential for therapy. Drug Resist Updat 2008;11:231-46.

10. Briggs J, Chamboredon S, Castellazzi M, et al. Transcriptional upregulation of SPARC, in response to c-Jun overexpression, contributes to increased motility and invasion of MCF7 breast cancer cells. Oncogene 2002;21:7077-91.

11. Wong SL, Sukkar MB. The SPARC protein: an overview of its role in lung cancer and pulmonary fibrosis and its potential role in chronic airways disease. Br J Pharmacol 2017;174:3-14.

12. Puolakkainen PA, Brekken RA, Muneer S, et al. Enhanced growth of pancreatic tumors in SPARC-null mice is associated with decreased deposition of extracellular matrix and reduced tumor cell apoptosis. Mol Cancer Res 2004;2:215-24.

13. Cheetham S, Tang MJ, Mesak F, et al. SPARC promoter hypermethylation in colorectal cancers can be reversed by 5-Aza-2'deoxycytidine to increase SPARC expression and 
improve therapy response. Br J Cancer 2008;98:1810-9.

14. DiMartino JF, Lacayo NJ, Varadi M, et al. Low or absent SPARC expression in acute myeloid leukemia with MLL rearrangements is associated with sensitivity to growth inhibition by exogenous SPARC protein. Leukemia 2006;20:426-32.

15. Ambros V. The functions of animal microRNAs. Nature 2004;431:350-5.

16. Dragomir MP, Knutsen E, Calin GA. SnapShot: Unconventional miRNA Functions. Cell 2018;174:10381038.e1.

17. Wang H, Zhu Y, Zhao M, et al. miRNA-29c suppresses lung cancer cell adhesion to extracellular matrix and metastasis by targeting integrin beta 1 and matrix metalloproteinase2 (MMP2). PLoS One 2013;8:e70192.

18. Fan Y, Song X, Du H, et al. Down-regulation of miR29c in human bladder cancer and the inhibition of proliferation in T24 cell via PI3K-AKT pathway. Med Oncol 2014;31:65.

19. Zhang JX, Mai SJ, Huang XX, et al. MiR-29c mediates epithelial-to-mesenchymal transition in human colorectal carcinoma metastasis via PTP4A and GNA13 regulation of beta-catenin signaling. Ann Oncol 2014;25:2196-204.

Cite this article as: Zou T, Gao Y, Qie M. MiR-29c-3p inhibits epithelial-mesenchymal transition to inhibit the proliferation, invasion and metastasis of cervical cancer cells by targeting SPARC. Ann Transl Med 2021;9(2):125. doi: 10.21037/atm-207272
20. Zhang S, Jin J, Tian X, et al. hsa-miR-29c-3p regulates biological function of colorectal cancer by targeting SPARC. Oncotarget 2017;8:104508-24.

21. Lv T, Jiang L, Kong L, et al. MicroRNA-29c-3p acts as a tumor suppressor gene and inhibits tumor progression in hepatocellular carcinoma by targeting TRIM31. Oncol Rep 2020;43:953-64.

22. Hu Z, Cai M, Zhang Y, et al. miR-29c-3p inhibits autophagy and cisplatin resistance in ovarian cancer by regulating FOXP1/ATG14 pathway. Cell Cycle 2020;19:193-206.

23. Chen G, Zhou T, Li Y, et al. p53 target miR-29c-3p suppresses colon cancer cell invasion and migration through inhibition of PHLDB2. Biochem Biophys Res Commun 2017;487:90-5.

24. Song H, Tao Y, Ni N, et al. miR-128 targets the CC chemokine ligand 18 gene (CCL18) in cutaneous malignant melanoma progression. J Dermatol Sci 2018;91:317-24.

25. Song H, Ding L, Zhang S, et al. MiR-29 family members interact with SPARC to regulate glucose metabolism. Biochem Biophys Res Commun 2018;497:667-74.

(English Language Editor: C. Betlazar-Maseh) 